\title{
Boosting the Seebeck Coefficient for Organic Coordination Polymers: Role of Doping-Induced Polaron Band Formation
}

\author{
Yunpeng Liu, Wen Shi, Tianqi Zhao, Dong Wang *, and Zhigang Shuai * \\ MOE Key Laboratory of Organic OptoElectronics and Molecular Engineering, Department of \\ Chemistry, Tsinghua University, Beijing 100084, PR China
}

\begin{abstract}
Organic polymers are becoming emerging thermoelectric materials. Tremendous progresses have been achieved for p-type doping, but efficient n-type organic materials are still rare. By investigating potassium-doped n-type poly(nickel-ethylenetetrathiolate) using density functional theory coupled with Boltzmann transport equation, we find that (i) formation of electron polaron band (EPB) split from conduction band (CB) dominates electron transport; (ii) at low doping concentration, the upper CB gets involved in transport in addition to EPB as temperature rises, leading to highly elevated Seebeck coefficient and power factor; (iii) at even higher temperature, since the CB starts to dominate, Seebeck coefficient levels off and then decreases with temperature. Such "exotic" non-monotonic temperature effect has been found in experiment but never been explained. We find such behavior is primarily due to polaron effect. Doping induced polaron band
\end{abstract}


can be employed to boost the Seebeck coefficient, making organic coordination polymer a peculiar n-type thermoelectric material.

\section{TOC Graphic}

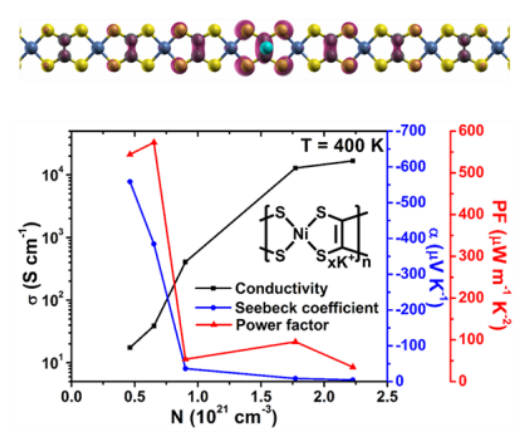

As a green energy solution to waste heat recycling, thermoelectrics has been gaining renewed attention. ${ }^{1-5}$ However, the low energy conversion efficiency has limited the application. ${ }^{3}$ The performance of thermoelectric materials is evaluated by the figure of merit $\mathrm{zT}=\frac{\alpha^{2} \sigma T}{\kappa_{e}+\kappa_{L}}$, where $\alpha$ is the Seebeck coefficient, $\sigma$ is the electrical conductivity, $T$ is the absolute temperature, $\kappa_{e}$ and $\kappa_{L}$ are electronic and lattice thermal conductivity, respectively. Therefore, the effective way to improve the performance of thermoelectric materials is to increase the Seebeck coefficient and conductivity of the material, and reduce the total thermal conductivity. Nevertheless, this is often challenging because these parameters are coupled with each other. ${ }^{1}$

The development of organic thermoelectric materials (OTEs) has advanced rapidly in recent years. Compared with inorganic materials, OTEs have the advantages of low cost, low toxicity, 
and low thermal conductivity. ${ }^{6}$ Owing to their low electrical conductivity, doping is usually needed to improve the thermoelectric performance. By virtue of the careful control of the doping level and removal of ineffective dopants, zT values of 0.25 and 0.42 have been achieved in tosylate (Tos) and polystyrene sulphonic acid (PSS) doped poly(3,4-ethylenedioxythiophene) (PEDOT), respectively. ${ }^{7-8}$ This makes PEDOT by far the best p-type OTE. The development of n-type OTEs also makes significant progress. The poly(nickel-ethylenetetrathiolate) (poly[Ni-ett]), a metal coordination polymer first synthesized by Poleschner et al.,${ }^{9}$ has been found to be a highperforming n-type OTE. ${ }^{10-14}$ In 2016 , zT of 0.32 was reported for the potassium-doped poly[Niett] prepared by electrochemical deposition, ${ }^{13}$ which is so far record-high among n-type OTEs.

However, understandings towards the role dopants played in optimizing the performance of OTEs are far from satisfactory. In contrast to inorganic thermoelectric materials, OTEs are soft and flexible, so the dopants not only inject charge carriers to the host materials, they may also affect the conduction of charge carriers, via altering the packing structure of the host and scattering with the charge carriers. Crispin et al. showed that the p-doping of PEDOT with Tos counterion altered the electronic structure of polymer via bipolaron formation. The formation of bipolaron band makes PEDOT:Tos a semimetal, which is the origin of the large Seebeck coefficient observed. ${ }^{15-16}$ Previously, we studied the effect of doping on the thermoelectric properties of PEDOT, ${ }^{17}$ by explicitly including the counterions Tos and their scattering to charge carriers in the model. The scattering, which arises from the screened Coulomb interactions between the charges on PEDOT and the counterions, has been ascertained to play a dominant role in the thermoelectric 
transport of PEDOT:Tos. Recent studies of thermoelectric coordination polymers by Yang et al. were based on the rigid band model. ${ }^{18-20}$ In this work, we utilize an explicit doping model to uncover the doping effect on the thermoelectric properties of potassium-doped n-type poly[Ni-ett]. We observed the significant band structure change owing to the polaron formation on the polymer chain in poly $\left[\mathrm{K}(\mathrm{Ni}-\mathrm{ett})_{n}\right]$ (short for $\mathrm{K}_{1} \mathrm{Ni}_{n}$ hereafter). The electron polaron band (EPB) split from the conduction band $(\mathrm{CB})$ shows much lower carrier mobility than $\mathrm{CB}$, and dominates the n-type transport for both electrical conductivity and Seebeck coefficient at low temperature. As the temperature rises, charge carriers in lightly-doped $\mathrm{K}_{1} \mathrm{Ni}_{14}$ and $\mathrm{K}_{1} \mathrm{Ni}_{20}$ can be thermally activated from EPB to CB, boosting Seebeck coefficient and power factor to anomalously large values. At further higher temperature, CB takes over and the Seebeck coefficient starts to decline. Such abnormal temperature behavior of Seebeck coefficient, previously observed in experiment, ${ }^{13}$ is discussed with the concept of transport entropy and has been attributed to the polaron formation in conducting polymers.

Since the intrachain electronic coupling constitutes the major electron conduction pathway of poly[Ni-ett $],{ }^{13,21}$ here we set up a one-dimensional model for the crystalline domain of the material, which was manifested to exist by the grazing-incidence X-ray diffraction (GIXRD) result. ${ }^{13}$ Structural optimizations and electronic structure calculations were then carried out using the Vienna Ab-initio Simulation Package $(\mathrm{VASP})^{22}$ with the $\mathrm{LDA}+\mathrm{U}(\mathrm{U}=6.04 \mathrm{eV})$ functional. ${ }^{23-24}$ The optimized cell length of pristine poly[Ni-ett] (along the polymer chain, $a$ axis) is $5.85 \AA$, which is very close to the experimental value of $5.95 \AA^{25}$ The optimized potassium-doped polymers, 
poly $\left[\mathrm{K}(\mathrm{Ni}-\mathrm{ett})_{n}\right]$ (short for $\mathrm{K}_{1} \mathrm{Ni}_{n}$ hereafter), show that the $\mathrm{K}$-atom is located on top of the C-C bond, which is in accordance with the structural model proposed by Vogt et al. based on the experimental analysis. ${ }^{26}$ The doping level is denoted by $1 / n$ and usually less than $100 \%,{ }^{12-13,25-28}$ so we take $n$ from 1 to 20 to represent various doping levels.

We identify a structural transformation of the polymer backbone after doping upon charge injection (Figure 1a). ${ }^{28}$ Around 0.88 electrons are transferred from potassium to the polymer backbone based on Bader's charge analysis. The C-S bond is elongated and C-C bond is shortened (Figure 1a). The structural transformation is only observed in bonds close to $\mathrm{K}^{+}$. Such localized change of bond length indicates the formation of polarons due to Coulomb interactions between charge on the polymer backbone and counter ion, $\mathrm{K}^{+}$, as reported in previous theoretical studies of other polymers including PEDOT, polythiophene and polypyrrole..$^{29-31}$ 
(a)

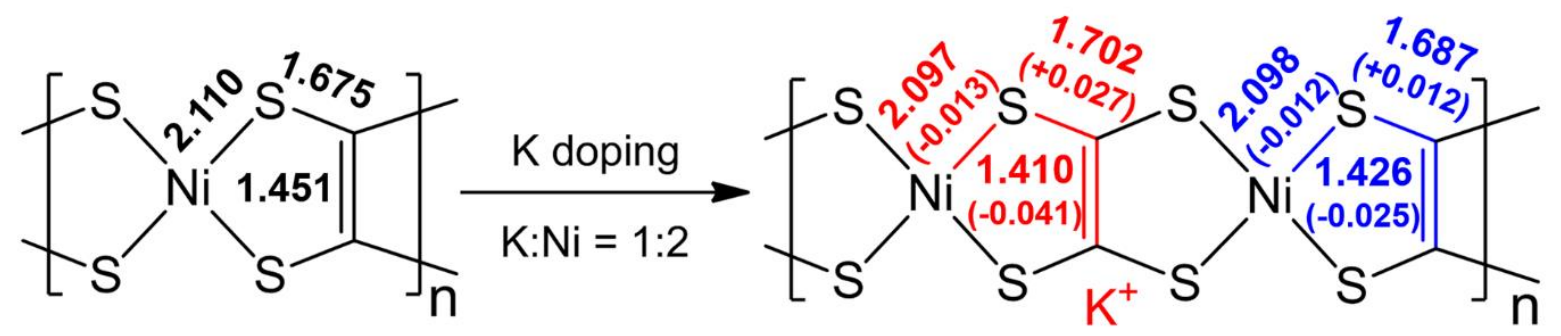

(b)

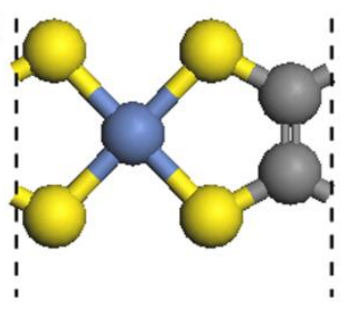

(c)
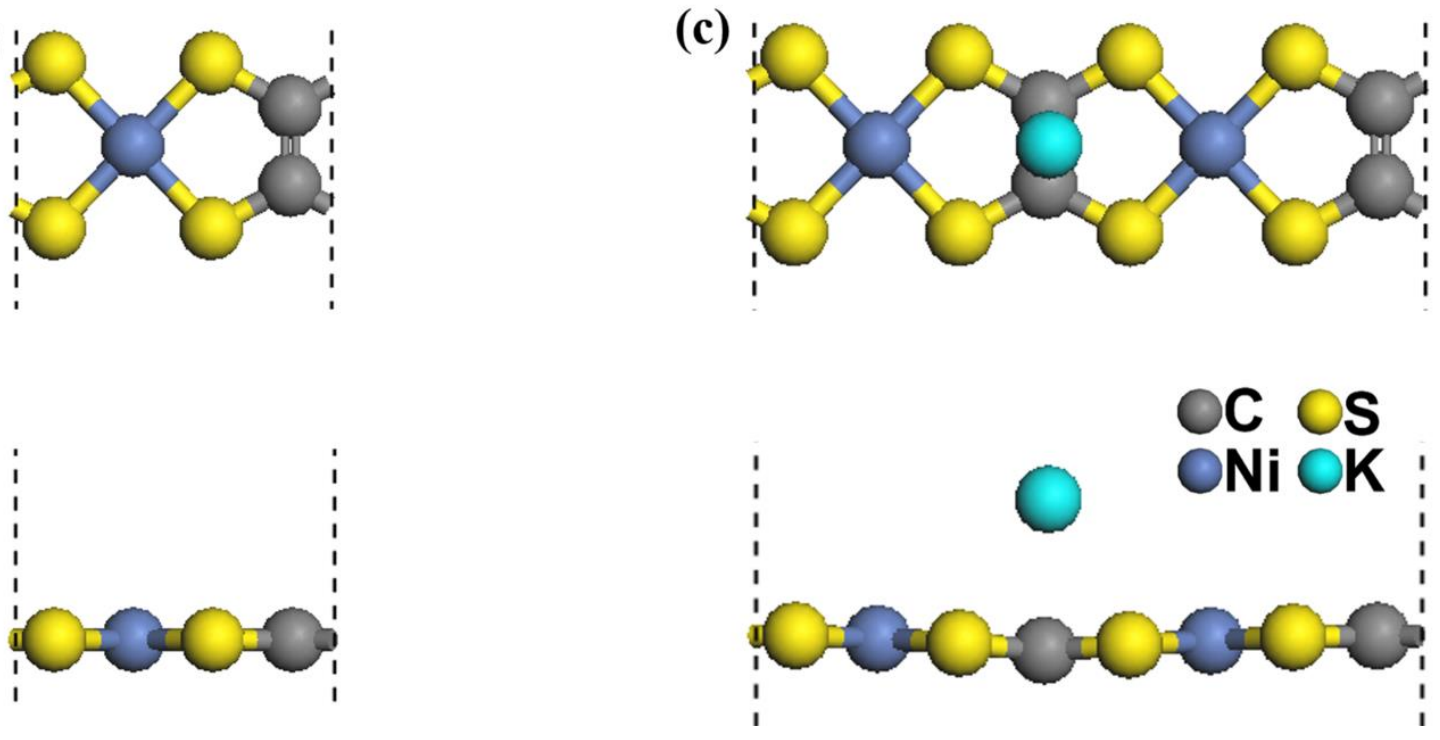

Figure 1. (a) Chemical structure of pristine poly[Ni-ett] and poly[K(Ni-ett)2]. The length of C-C, C-S, and Ni-S bonds (in unit of $\AA$ ) are given. The bond length change (in unit of $\AA$ ) after doping (in poly[K(Ni-ett) 2$]$ ) is shown in the parentheses as well. (b) Top and side views of optimized pristine poly[Ni-ett] in a unit cell. (c) Top and side views of poly[K(Ni-ett)2] in a unit cell. The color code for atoms is grey for $\mathrm{C}$, yellow for $\mathrm{S}$, blue for $\mathrm{Ni}$, and cyan for $\mathrm{K}$.

The pristine poly[Ni-ett] is a semiconductor with the direct band gap of $0.42 \mathrm{eV}$ at the $\Gamma$ point, with the conduction band width of $1.32 \mathrm{eV}$ (Figure 2a). The partial density of states (pDOS) shows 
that $p$ orbitals of $\mathrm{C}, \mathrm{S}$, and $\mathrm{Ni}$ as well as $d$ orbitals of Ni constitute the CB (Figure 2a), forming a $\pi$ - $d$ conjugation system. After K-doping, the CB splits into a series of narrow bands, and bandwidth of the lowest one decreases dramatically with increasing the numebr of nickel atoms $n$ (Figure 2e). According to pDOS (Figure 2a-d), the composition of bands barely changes after splitting, indicating that the band narrowing in doped polymers is not caused by the participation of dopant orbitals. Actually, $\mathrm{K}$ orbitals do not contribute to these bands. The electron density distribution at the Fermi level, as shown in Figure 2f, clearly demonstrates the charge localization near $\mathrm{K}^{+}$in lightly-doped polymers, such as $\mathrm{K}_{1} \mathrm{Ni}_{10}$. It is coincident with the localized bond distortion mentioned above, indicating the formation of polarons. According to previous theoretical and experimental researches, the polaron arises from both the electron-phonon coupling (often manifested by backbone distortion) and Coulomb interaction between the excess charge and the dopant through the "pinning effect", ${ }^{29,32-38}$ which lower the energy of charge carriers. The carriers become self-trapped and polarons are formed when the stabilization energy is large enough. ${ }^{36}$. The electron polaron band (EPB) arises in the forbidden band with the narrow band width due to selftrapping. ${ }^{38}$ The EPB here is half-filled with the Fermi energy lying in the band, which is a marked feature of polaron bands. The other bands split from the pristine CB are normal conduction bands (CBs), which possess better transport properties than EPB. The energy gap between EPB and the lowest $\mathrm{CB}$ is not large, giving the electrons in $\mathrm{EPB}$ a good chance to be thermally activated to $\mathrm{CB}$.

The exact size of polaron, or the charge localization length, can be derived from the inverse participation ratio (IPR), ${ }^{39}$ defined as 


$$
\operatorname{IPR}_{i}=\frac{\sum_{j}\left|c_{i j}\right|^{4}}{\left(\sum_{j}\left|c_{i j}\right|^{2}\right)^{2}}
$$

where $c_{i j}$ denotes the wave function expansion coefficient at site $j$ for the $i$-th crystal orbital. If the wave function is delocalized completely over $M$ sites, IPR $=1 / \mathrm{M}$. So the localization length is represented by $1 / \mathrm{IPR}$, and shown in Figure 2e for $\mathrm{K}_{1} \mathrm{Ni}_{n}$. With $n$ increasing, it converges to 4.5 monomers. The above analysis reveals a polaron size of 4.5 monomer sites. Polaron band width decreases exponentially with $n$ (Figure 2e), because the polaron coupling (hopping integral) decreases exponentially with the inter-polaron distance. 
(a)

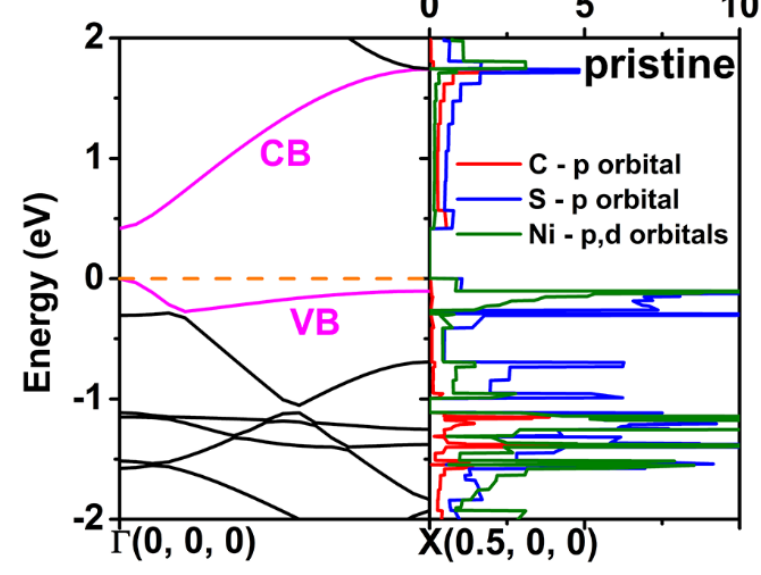

(c)

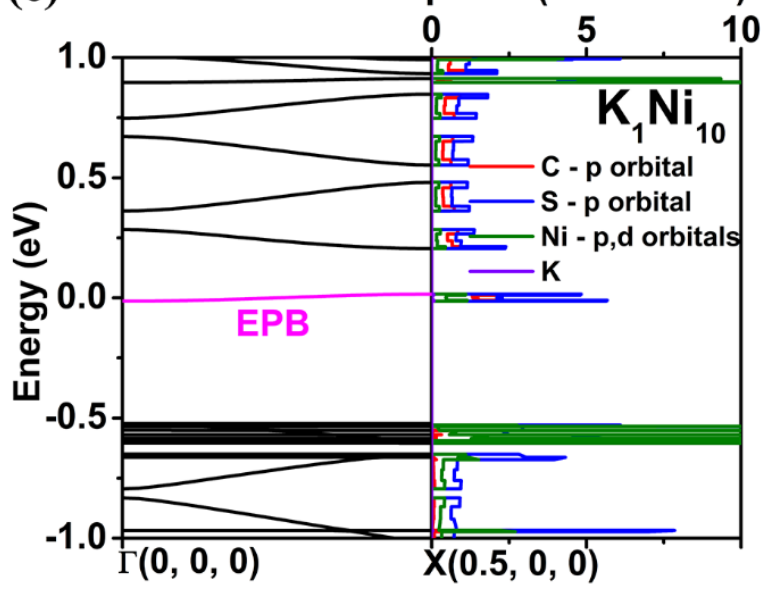

(e)

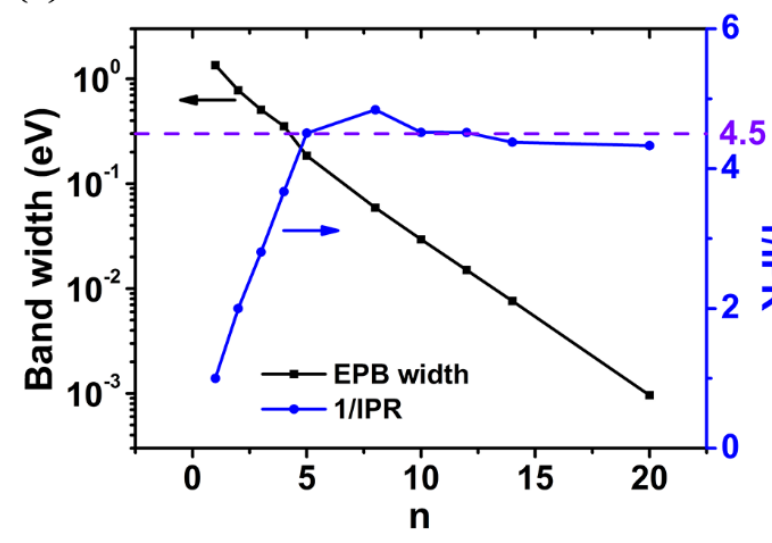

(b)

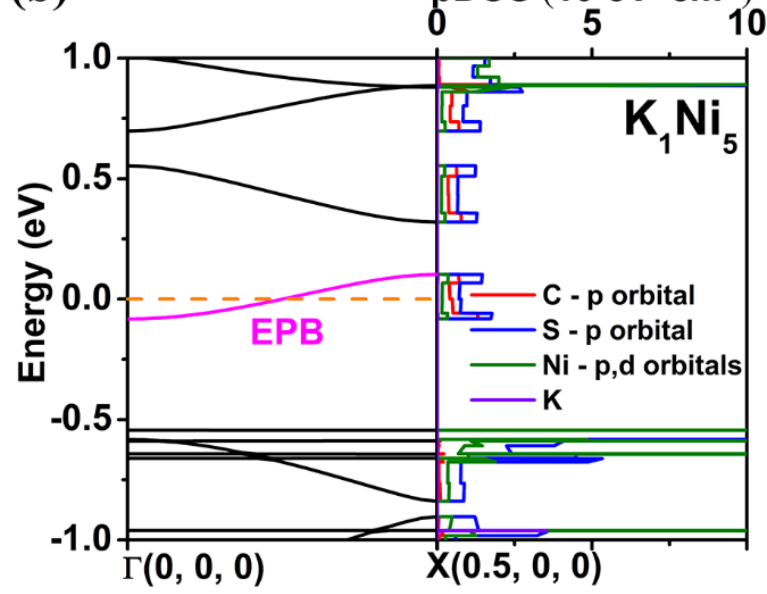

(d)

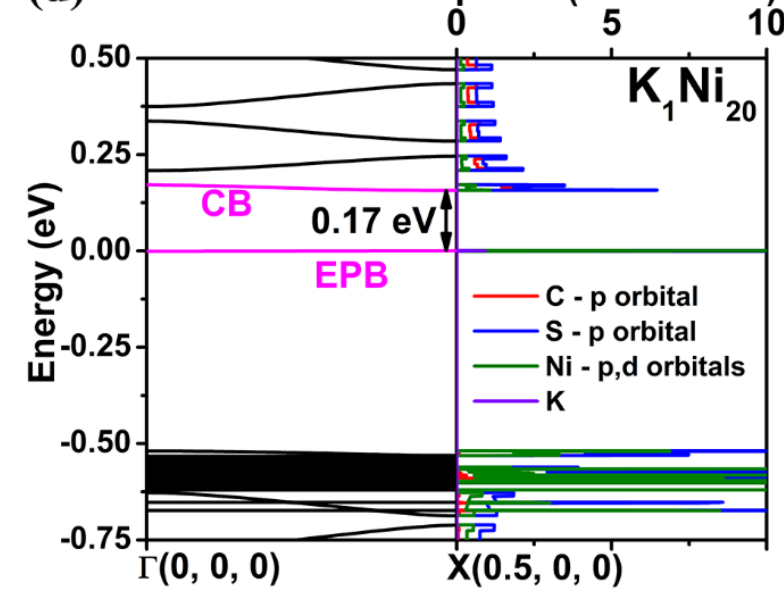

(f)

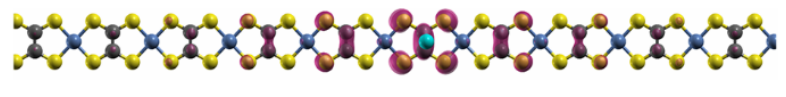

穴

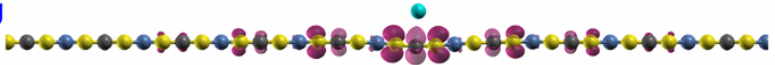

Figure 2. Band structure and partial density of states (pDOS) of (a) pristine poly[Ni-ett], (b)

$\mathrm{K}_{1} \mathrm{Ni}_{5}$, (c) $\mathrm{K}_{1} \mathrm{Ni}_{10}$ and (d) $\mathrm{K}_{1} \mathrm{Ni}_{20}$. The conduction band (CB) and valence band (VB) in the pristine poly[Ni-ett], the electron polaron band (EPB) in the doped polymers, as well as the 
lowest $\mathrm{CB}$ in $\mathrm{K}_{1} \mathrm{Ni}_{20}$ are highlighted in pink. The Fermi level is at $\mathrm{O} \mathrm{eV}$. (e) Electron polaron band width (EPBW) and charge localization length estimated by 1/IPR as a function of $\boldsymbol{n}$ for poly $\left[\mathrm{K}(\mathrm{Ni} \text {-ett) })_{n}\right]$. (f) Charge density distribution (violet red isosurface, top and side views) of EPB in poly[K(Ni-ett) $\left.{ }_{10}\right]$, which shows obvious charge localization near $\mathrm{K}^{+}$.

The polaron band narrowing and charge localization effect significantly have strong influences on the thermoelectric properties. The electrical conductivity $\sigma$ and the Seebeck coefficient $\alpha$ at the temperature of $400 \mathrm{~K}$ are shown in Figure 3 . The effective cross-sectional area of $5.9 \AA \times 3.2 \AA$ taken from experiment ${ }^{13}$ is applied to convert the conductance to conductivity. The Seebeck coefficients for $\mathrm{K}_{1} \mathrm{Ni}_{14}$ and $\mathrm{K}_{1} \mathrm{Ni}_{20}$ are substantially larger than other polymers. Although the electrical conductivity in lightly doped polymers is low due to the band narrowing and charge localization effect, the power factor reaches the peak value at the doping level of $n=14$. The conductivity and Seebeck coefficient relation obviously deviates from that derived from the oneband transport model, $\alpha \propto \ln \sigma$ (Figure S5). ${ }^{40}$ Herein, we propose a two-band transport model to explain the deviation and the temperature dependence of thermoelectric properties in lightly doped polymers. 


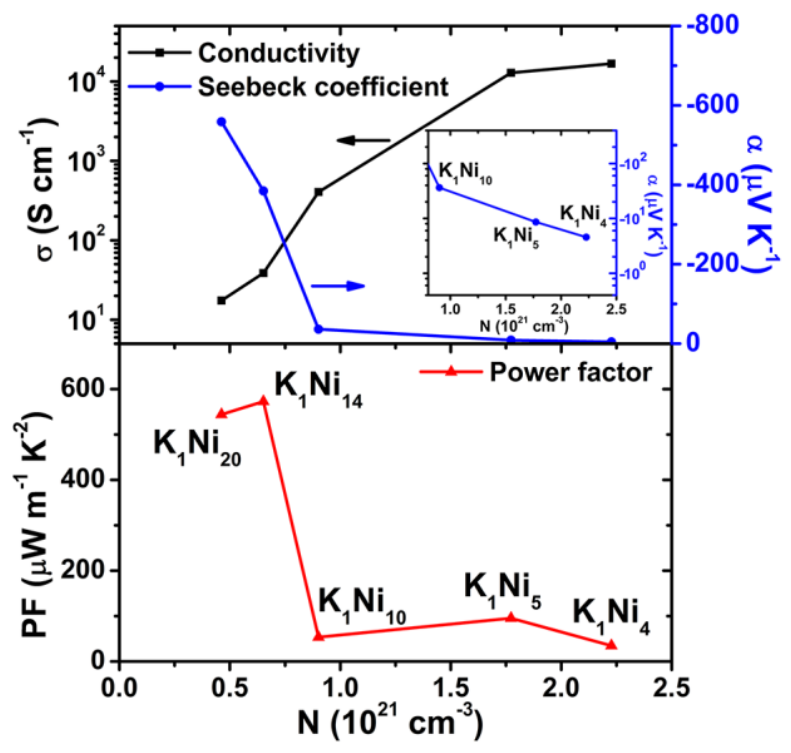

Figure 3. The conductivity, Seebeck coefficient, and power factor as a function of charge density $\mathrm{N}$ (at temperature $\mathrm{T}=\mathbf{4 0 0} \mathrm{K}$ ).

Figure 4 shows the charge mobility, conductivity, and Seebeck coefficient for $\mathrm{K}_{1} \mathrm{Ni}_{n}$ as a function of temperature. Two categories are easily demonstrated: those of heavy dopings with $n \leq$ 5 exhibit slight temperature dependence, and those of light dopings with $n=14$ and 20 show nonmonotonic temperature dependence, which is unusual and will be explained by including both EPB and $\mathrm{CB}$ in charge transport. The turnover in the conductivity-temperature curve (Figure 4a) was observed in experiment on electrochemical doped poly[ $\mathrm{K}_{x}(\mathrm{Ni}$-ett $\left.)\right] \cdot{ }^{13}$. In lightly doped polymers, there exists a small energy gap between EPB and CB. When temperature is low, EPB dominates charge transport, which gives rise to low mobility and conductivity. With the increase of temperature, the ionized impurity scattering, the dominant scattering mechanism here (Figure S6), 
is enhanced due to the decrease of screening strength (Figure S7), which then leads to the reduction of mobility and conductivity (Figure $4 \mathrm{a}$ and $4 \mathrm{~b}$ ). However, as temperature rises further, more charge carriers can be thermally activated from EPB to CB due to the small energy gap between them (e.g. the energy gap is $\sim 0.17 \mathrm{eV}$ in $\mathrm{K}_{1} \mathrm{Ni}_{20}$ ). Since $\mathrm{CB}$ (e.g. the bandwidth is $14.6 \mathrm{meV}$ in $\mathrm{K}_{1} \mathrm{Ni}_{20}$ ) is much more dispersed than EPB (e.g. the bandwidth is $0.96 \mathrm{meV}$ in $\mathrm{K}_{1} \mathrm{Ni}_{20}$ ), charge carriers in $\mathrm{CB}$ move faster. So at higher temperature, both mobility and conductivity increase.

(a)

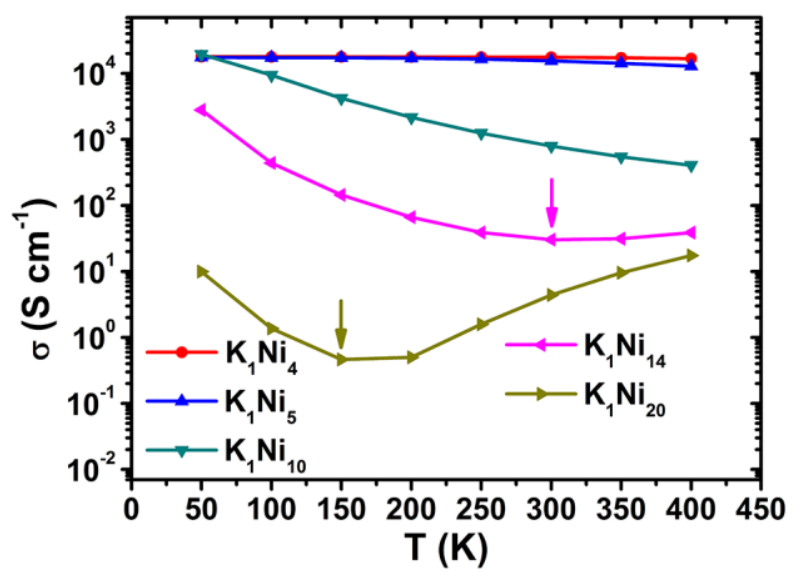

(c)

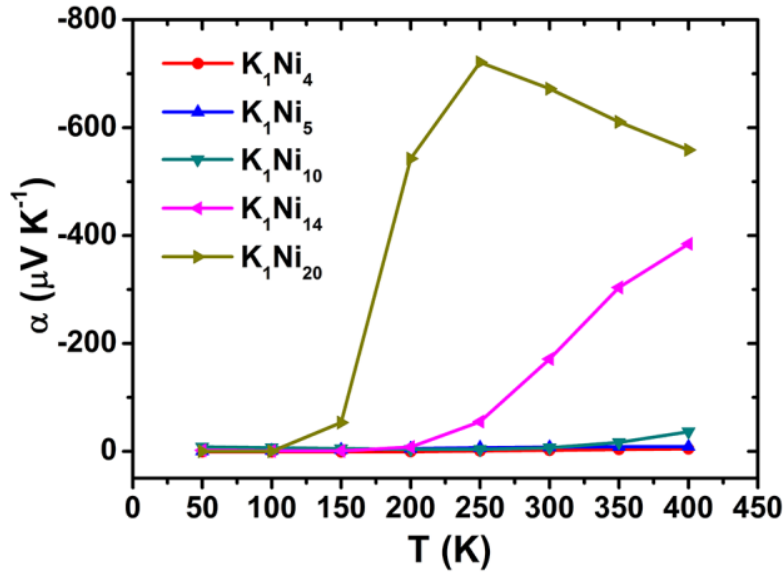

(b)

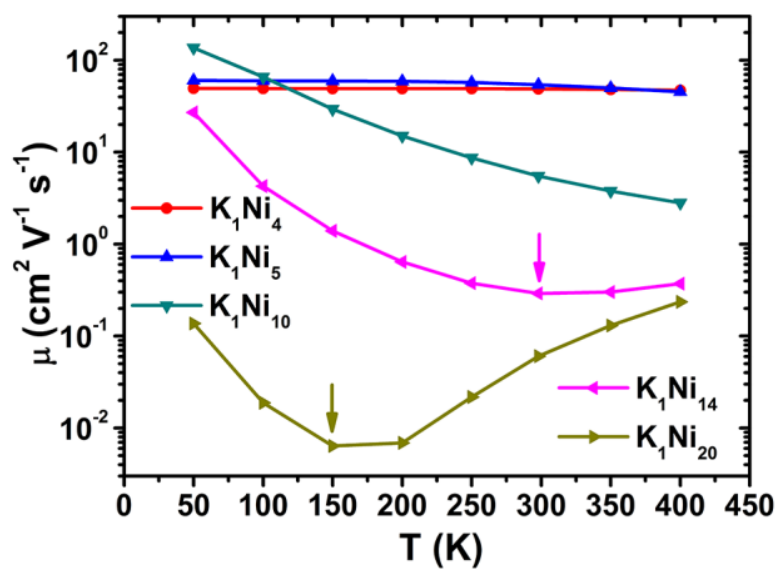

(d)

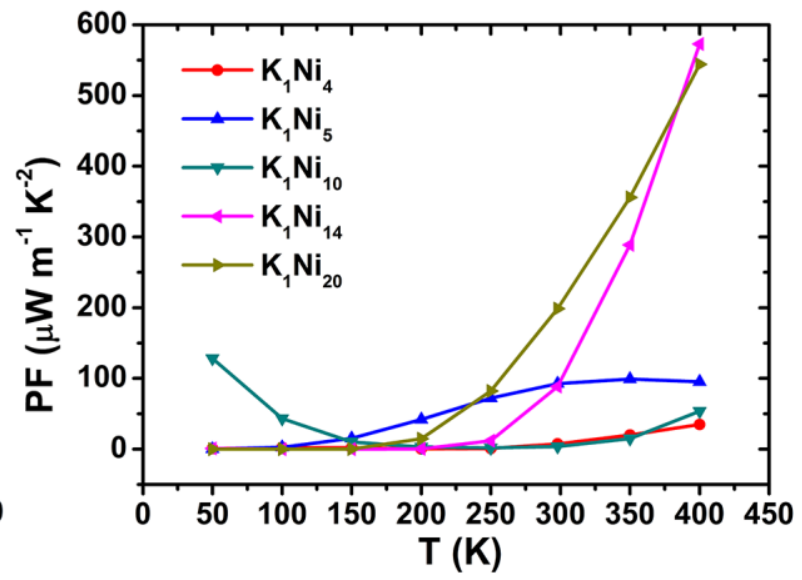


Figure 4. (a) Electric conductivity, (b) mobility, (c) Seebeck coefficient, and (d) power factor (PF) as a function of temperature for poly $\left[\mathrm{K}(\mathrm{Ni} \text {-ett })_{n}\right]$ at different doping levels. The turning points of electrical conductivity and mobility in $\mathrm{K}_{1} \mathrm{Ni}_{14}$ and $\mathrm{K}_{1} \mathrm{Ni}_{20}$ are specifically denoted in the figure.

The sudden increases in Seebeck coefficient at $250 \mathrm{~K}$ and $150 \mathrm{~K}$ are found in $\mathrm{K}_{1} \mathrm{Ni}_{14}$ and $\mathrm{K}_{1} \mathrm{Ni}_{20}$, respectively (Figure $4 \mathrm{c}$ ), which coincide with the turning points observed in the conductivity-temperature curve, indicating that $\mathrm{CB}$ starts to play a role in charge transport. Surprisingly, the Seebeck coefficient of $\mathrm{K}_{1} \mathrm{Ni}_{20}$ starts to drop again at $250 \mathrm{~K}$ (Figure 4c). According to our calculation, such nonmonotonic temperature dependence of Seebeck coefficient is due to the two-band transport behavior (see Figure 5 and the corresponding below). Such unusual behavior has been observed in experiment, where the Seebeck coefficient increases with temperature first and then starts to drop at $510 \mathrm{~K}$ in electrochemical doped poly[ $\mathrm{K}_{x}(\mathrm{Ni}$-ett $\left.)\right] .{ }^{13}$

According to the Onsager's reciprocal relations and Kelvin relations, the Seebeck coefficient $\alpha$ can be expressed as the "transport entropy" $S$ divided by the charge of electron $-e{ }^{41}$ This transport entropy consists of three parts: the change of entropy of mixing upon adding a carrier, the change of entropy resulting from the spin degeneracy, and the change of entropy due to the effect of injecting a carrier on molecular vibrations. ${ }^{38,42}$ Since the last two terms are not sensitive to the temperature, ${ }^{38,42}$ only the change of entropy of mixing is considered when discussing the temperature effect. In the narrow band limit, the entropy of mixing for a system with $N_{0}$ states and $N=N_{0} f_{0}$ carriers (where $f_{0}$ is the Fermi-Dirac distribution function) can be expressed as 


$$
S_{m i x}=-N_{0} k_{\mathrm{B}}\left[f_{0} \ln \left(f_{0}\right)+\left(1-f_{0}\right) \ln \left(1-f_{0}\right)\right]
$$

And the corresponding Seebeck coefficient is

$$
\alpha_{\text {mix }}=-\frac{1}{e} \frac{\partial S_{\text {mix }}}{\partial N}=-\frac{1}{e} \frac{\partial S_{\text {mix }}}{\partial\left(N_{0} f_{0}\right)}=-\frac{k_{\mathrm{B}}}{e} \ln \left(\frac{1-f_{0}}{f_{0}}\right)
$$

Obviously, the Seebeck coefficient is large when the transport band is nearly empty or nearly fullfilled. For a half-filled band $f_{0}=0.5$, and $\alpha_{m i x}=0$. At low temperature, the EPB of $\mathrm{K}_{1} \mathrm{Ni}_{14}$ and $\mathrm{K}_{1} \mathrm{Ni}_{20}$ is narrow and half-filled, so the Seebeck coefficient is small. The conclusion that polaron bands have low Seebeck coefficient was also drawn by Bubnova et al. ${ }^{15}$ The sudden increase of Seebeck and anomalously large values at higher temperature can be attributed to the thermal activation of electrons from EPB to CB. Since CB is now nearly empty, its Seebeck coefficient is large. Our conclusion that the wide CB possesses larger Seebeck coefficient than the narrow EPB is not in conflict with Mahan et al.'s viewpoint that narrow band benefits the thermoelectric conversion ${ }^{43}$ because their deduction is based on the assumption that the two bands have similar electron occupancy $f_{0}$, which is however very different for CB and EPB here. The occupancy of the band is more important to the Seebeck coefficient in our case. In the regime of two-band transport, the total Seebeck coefficient $\alpha$ is the average of $\alpha_{i}(i=\mathrm{EPB}, \mathrm{CB})$ weighted by their contribution $\sigma_{i}$ to the total conductivity: ${ }^{38}$

$$
\alpha=\frac{\alpha_{E P B} \sigma_{E P B}+\alpha_{C B} \sigma_{C B}}{\sigma_{E P B}+\sigma_{C B}}
$$

With more carriers activated to $\mathrm{CB}$ at elevated temperature, both $\sigma_{\mathrm{CB}}$ and $\alpha$ increase. 
Yet the Seebeck coefficient cannot keep rising. When $\mathrm{CB}$ dominates the charge transport ( $\sigma_{\mathrm{CB}}$ )> $\sigma_{\mathrm{EPB}}$ ), Eq. (4) is reduced to $\alpha=\alpha_{\mathrm{CB}}$, which decreases with temperature and $f_{0}$. This explains the drop of $\alpha$ when $T>250 \mathrm{~K}$ in $\mathrm{K}_{1} \mathrm{Ni}_{20}$.

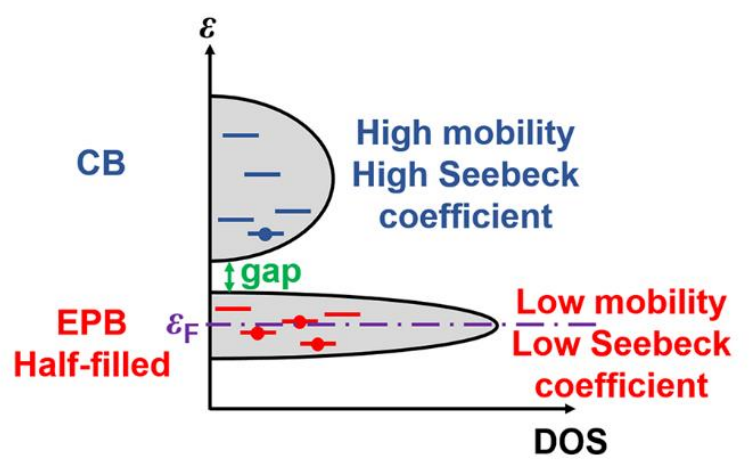

Figure 5. Schematics of the two-band transport model for K-doped poly[Ni-ett]. The Fermi level $\varepsilon_{F}$ lies in the half-filled electron polaron band (EPB). Electrons can be thermally activated from EPB to conduction band (CB) at high temperature due to the small energy

gap.

Overall, the two-band transport model has satisfactorily explained the temperature dependence of thermoelectric properties in lightly doped polymers, highlighting the importance of polaron induced charge localization effect in boosting Seebeck coefficient of $\mathrm{K}_{1} \mathrm{Ni}_{n}$.

To conclude, we have identified the polaron formation in K-doped poly[Ni-ett]. The polaron size is of $\sim 4.5$ monomers in the vicinity of $\mathrm{K}^{+}$, thus it has been directly observed in lightly doped polymers and has changed significantly thermoelectric transport behavior. The polaron induced charge localization causes the significant narrowing of EPB, and dramatically reduces the 
conductivity. Doping can reduce the energy gap between half-filled EPB and CB, making thermal activation of charge carriers to the much wider CB feasible at higher temperatures. The unusual non-monotonic temperature dependence of Seebeck coefficient and its sudden increase for lightly doped $\mathrm{K}_{1} \mathrm{Ni}_{n}$ with $n=14$ and 20 can be perfectly explained by the polaron band formation coupled with a two-band transport model. The calculated optimal doping level is $1 / \mathrm{n}=1 / 14$ at $400 \mathrm{~K}$, which is close to the value $(\sim 10 \%)$ found in experiment. ${ }^{12}$

\section{Computational details}

Electronic structure calculation. $\Gamma$-centered k-meshes of $4 \times 1 \times 1$ (pristine and poly[K(Ni-ett) $\left.{ }_{n}\right]$ with $n=1,2,3), 2 \times 1 \times 1(n=4)$, and $1 \times 1 \times 1(n=5,8,10,12,14,20)$ were used during optimization, while $8 \times 1 \times 1$ (pristine and $n=1,2,3,4), 4 \times 1 \times 1(n=5)$, and $2 \times 1 \times 1(n=8,10,12,14,20)$ for single-point energy and charge density calculations. Band energies on fine Monkhorst-Pack kmeshes of $300 \times 1 \times 1\left(\mathrm{~K}_{1} \mathrm{Ni}_{4}\right), 240 \times 1 \times 1\left(\mathrm{~K}_{1} \mathrm{Ni}_{5}\right), 120 \times 1 \times 1\left(\mathrm{~K}_{1} \mathrm{Ni}_{10}\right), 80 \times 1 \times 1\left(\mathrm{~K}_{1} \mathrm{Ni}_{14}\right), 60 \times 1 \times 1$ $\left(\mathrm{K}_{1} \mathrm{Ni}_{20}\right)$ were used for BoltzTraP calculation. ${ }^{44}$ Band energy interpolation of 50 times was applied for all systems.

Relaxation time calculation. The electrical conductivities and Seebeck coefficients were calculated based on the Boltzmann transport equation ${ }^{45}$ through BoltzTraP code $^{44}$. The relaxation time is obtained through first-principles calculations. Both acoustic phonon scattering and ionized impurity scattering mechanisms were included to account for the charge carrier relaxation, with the former modeled by the deformation potential (DP) theory ${ }^{46}$ and the latter derived from the 
Lindhard screening function for Coulomb interaction between the charge carrier and the counterion. ${ }^{47-48}$ Assuming that the scatterings are independent, the Matthiessen's rule was applied to get the total relaxation time: $\tau^{-1}=\tau_{\mathrm{ac}}^{-1}+\tau_{\text {ion }}^{-1}$, where $\tau_{\mathrm{ac}}$ and $\tau_{\text {ion }}$ are relaxation times due to acoustic phonon scattering and ionized impurity scattering, respectively. The acoustic phonon relaxation time is obtained by

$$
\frac{1}{\tau_{\mathrm{k}, \mathrm{ac}}}=\frac{2 \pi}{\hbar} \sum_{\mathrm{k}^{\prime}} \frac{k_{\mathrm{B}} T E_{1}^{2}}{C_{a}^{\mathrm{ID}}} \delta\left(\varepsilon_{\mathrm{k}}-\varepsilon_{\mathrm{k}^{\prime}}\right)\left(1-\frac{v_{\mathrm{k}^{\prime}}}{v_{\mathrm{k}}}\right)
$$

where $\varepsilon_{\mathrm{k}}$ and $v_{\mathrm{k}}$ are the energy and group velocity of electronic state $|\mathrm{k}\rangle$, respectively. $E_{1}$ is the deformation potential constant, and $C_{a}{ }^{1 \mathrm{D}}$ the $1-\mathrm{D}$ elastic constant along the polymer chain $(a$ direction).

The ionized impurity relaxation time is obtained by

$$
\frac{1}{\tau_{\mathrm{k}, \text { ion }}}=\frac{2 \pi}{\hbar} \sum_{\mathrm{k}^{\prime}}\left|\frac{V_{\mathrm{e}-\text { ion }}(\mathrm{q})}{1+S c r \cdot F(\mathrm{q})}\right|^{2} \delta\left(\varepsilon_{\mathrm{k}}-\varepsilon_{\mathrm{k}^{\prime}}\right)\left(1-\frac{v_{\mathrm{k}^{\prime}}}{v_{\mathrm{k}}}\right)
$$

where

$$
\begin{gathered}
V_{\text {e-ion }}(\mathrm{q})=-\frac{Z e^{2}}{2 \pi \varepsilon_{r} \varepsilon_{0} L_{0}} \iint \mathrm{d} y \mathrm{~d} z K_{0}\left(|\mathrm{q}| \sqrt{\left(y-y_{0}\right)^{2}+\left(z-z_{0}\right)^{2}}\right) \rho(y, z) \\
F(\mathrm{q}) \equiv \iiint \int \mathrm{d} y \mathrm{~d} y^{\prime} \mathrm{d} z \mathrm{~d} z^{\prime} \mathrm{K}_{0}\left(|\mathrm{q}| \sqrt{\left(y-y^{\prime}\right)^{2}+\left(z-z^{\prime}\right)^{2}}\right) \rho(y, z) \rho\left(y^{\prime}, z^{\prime}\right) \\
S c r=\frac{\gamma e^{2} n_{1 \mathrm{D}, e / \mathrm{h}}}{2 \pi \varepsilon_{r} \varepsilon_{0} k_{\mathrm{B}} T}
\end{gathered}
$$

Here the screening effect caused by the free carriers is considered. $V_{\text {e-ion }}(q)$ is the unscreened scattering matrix. $F(\mathrm{q})$ describes the influence of wave-vector change $\mathrm{q}$ of charge carriers during 
the scattering on the screening strength. The screening factor $S c r$ reflects the effect of carrier concentration and temperature on the screening strength. $\left(y_{0}, z_{0}\right)$ and $Z$ respectively are the coordinate and charge number of the ionized impurity. $e$ is the elementary charge. $\varepsilon_{r}$ is the relative dielectric constant and $\varepsilon_{0}$ the permittivity of vacuum. $L_{0}$ is the unit cell length. $K_{0}$ is the zeroth-

order modified Bessel function of the second kind. $\rho(y, z)=|\chi(y, z)|^{2}$ is the charge density distribution in the plane perpendicular to the chain, and $\chi(y, z)$ represents the wave function in the plane perpendicular to the chain. $n_{1 \mathrm{D}, e / h}$ is the $1 \mathrm{D}$ concentration of electrons/holes. The factor $\gamma=\frac{g_{\mathrm{s}}}{L_{0}} \int_{-\pi / L_{0}}^{\pi / L_{0}} f_{0}\left(1-f_{0}\right) \frac{L_{0}}{2 \pi} \mathrm{dk} / n_{1 \mathrm{D}, e / \mathrm{h}}$, where $g_{s}$ represents spin degeneracy. A detailed derivation of the ionized impurity relaxation time formula is provided in Supporting Information.

The deformation potential constant was obtained by the linear fit of the Fermi level shift with the lattice dilation, calibrated by the vacuum level. The Bader charge analysis was carried out to get the charge carried by the ionized potassium. ${ }^{49}$ The charge on potassium had a similar value of about +0.88 in all doped chains, showing a nearly complete charge transfer (Figure S4). We also calculated the relative dielectric constant $\varepsilon_{r}$ of the pristine chain using the VASP software, which is 4.37. The ionic charge and dielectric constant were used for the calculation of ionized impurity scattering time.

\section{Acknowledgements}

This work was supported by the National Natural Science Foundation of China (Grant No. 21788102 and 21673123) and the Ministry of Science and Technology of China (Grant No. 
2017YFA0204501 and 2015CB655002). Computational resources are provided by the Tsinghua

Supercomputing Center.

Supporting Information Available: Band structure, pDOS, charge density of EPB, deformation potential, elastic constant, and Bader's charge analysis for K-doped poly[K(Ni-ett) $]$; conductivity and Seebeck coefficient relationship; derivation of the 1-D ionized impurity scattering matrix element; temperature-dependent screening in ionized impurity scattering.

\section{References}

(1) Snyder, G. J.; Toberer, E. S. Complex thermoelectric materials. Nat. Mater. 2008, 7, 105-114.

(2) DiSalvo, F. J. Thermoelectric cooling and power generation. Science 1999, 285, 703-706.

(3) Bell, L. E. Cooling, heating, generating power, and recovering waste heat with thermoelectric systems. Science 2008, 321, 1457-1461.

(4) Heremans, J. P. Thermoelectricity: The ugly duckling. Nature 2014, 508, 327-328.

(5) He, J.; Tritt, T. M. Advances in thermoelectric materials research: Looking back and moving forward. Science 2017, 357, eaak9997.

(6) Zhang, Q.; Sun, Y.; Xu, W.; Zhu, D. Organic thermoelectric materials: emerging green energy materials converting heat to electricity directly and efficiently. Adv. Mater. 2014, 26, 6829-6851.

(7) Kim, G. H.; Shao, L.; Zhang, K.; Pipe, K. P. Engineered doping of organic semiconductors for enhanced thermoelectric efficiency. Nat. Mater. 2013, 12, 719-723.

(8) Bubnova, O.; Khan, Z. U.; Malti, A.; Braun, S.; Fahlman, M.; Berggren, M.; Crispin, X. Optimization of the thermoelectric figure of merit in the conducting polymer poly(3,4-ethylenedioxythiophene). Nat. Mater. 2011, 10, 429-433.

(9) Poleschner, H.; John, W.; Hoppe, F.; Fanghänel, E.; Roth, S. Tetrathiafulvalene. XIX. Synthese und Eigenschaften elektronenleitender Poly-Dithiolenkomplexe mit Ethylentetrathiolat und Tetrathiafulvalentetrathiolat als Brückenliganden. J. Prakt. Chem. 1983, 325, 957-975.

(10) Sun, Y.; Sheng, P.; Di, C.; Jiao, F.; Xu, W.; Qiu, D.; Zhu, D. Organic thermoelectric materials and devices based on p- and n-type poly(metal 1,1,2,2-ethenetetrathiolate)s. Adv. Mater. 2012, 24, 932-937.

(11) Jiao, F.; Di, C. A.; Sun, Y. M.; Sheng, P.; Xu, W.; Zhu, D. B. Inkjet-printed flexible organic thin-film thermoelectric devices based on p- and n-type poly(metal 1,1,2,2-ethenetetrathiolate)s/polymer composites through ball-milling. Philos. T. R. Soc. A 2014, 372, 20130008.

(12) Sun, Y. H.; Zhang, J. J.; Liu, L. Y.; Qin, Y. K.; Sun, Y. M.; Xu, W.; Zhu, D. B. Optimization of the thermoelectric properties of poly(nickel-ethylenetetrathiolate) synthesized via potentiostatic deposition. Sci. China Chem. 2016, 59, 1323-1329.

(13) Sun, Y.; Qiu, L.; Tang, L.; Geng, H.; Wang, H.; Zhang, F.; Huang, D.; Xu, W.; Yue, P.; Guan, Y. S.; Jiao, F.; Sun, 
Y.; Tang, D.; Di, C. A.; Yi, Y.; Zhu, D. Flexible n-Type High-Performance Thermoelectric Thin Films of Poly(nickelethylenetetrathiolate) Prepared by an Electrochemical Method. Adv. Mater. 2016, 28, 3351-3358.

(14) Menon, A. K.; Uzunlar, E.; Wolfe, R. M. W.; Reynolds, J. R.; Marder, S. R.; Yee, S. K. Metallo-organic n-type thermoelectrics: Emphasizing advances in Nickel-ethenetetrathiolates. J. Appl. Polym. Sci. 2017, 134, 44402.

(15) Bubnova, O.; Khan, Z. U.; Wang, H.; Braun, S.; Evans, D. R.; Fabretto, M.; Hojati-Talemi, P.; Dagnelund, D.; Arlin, J. B.; Geerts, Y. H.; Desbief, S.; Breiby, D. W.; Andreasen, J. W.; Lazzaroni, R.; Chen, W. M.; Zozoulenko, I.; Fahlman, M.; Murphy, P. J.; Berggren, M.; Crispin, X. Semi-metallic polymers. Nat. Mater. 2014, 13, 190-194.

(16) Chabinyc, M. Thermoelectric polymers: Behind organics' thermopower. Nat. Mater. 2014, 13, 119-121.

(17) Shi, W.; Zhao, T. Q.; Xi, J. Y.; Wang, D.; Shuai, Z. G. Unravelling Doping Effects on PEDOT at the Molecular Level: From Geometry to Thermoelectric Transport Properties. J. Am. Chem. Soc. 2015, 137, 12929-12938.

(18) Yong, X.; Shi, W.; Wu, G.; Goh, S. S.; Bai, S.; Xu, J.-W.; Wang, J.-S.; Yang, S.-W. Tuning the thermoelectric performance of $\pi-\mathrm{d}$ conjugated nickel coordination polymers through metal-ligand frontier molecular orbital alignment. J. Mater. Chem. A 2018, 6, 19757-19766.

(19) Shi, W.; Wu, G.; Yong, X.; Deng, T.; Wang, J. S.; Zheng, J. C.; Xu, J.; Sullivan, M. B.; Yang, S. W. OrbitalEngineering-Based Screening of $\pi$-Conjugated $\mathrm{d}^{8}$ Transition-Metal Coordination Polymers for High-Performance $\mathrm{n}$ Type Thermoelectric Applications. ACS Appl. Mater. Interfaces 2018, 10, 35306-35315.

(20) Shi, W.; Wu, G.; Hippalgaonkar, K.; Wang, J. S.; Xu, J.; Yang, S. W. Poly(nickel-ethylenetetrathiolate) and Its Analogs: Theoretical Prediction of High-Performance Doping-Free Thermoelectric Polymers. J. Am. Chem. Soc. 2018, 140, 13200-13204.

(21) Alvarez, S.; Vicente, R.; Hoffmann, R. Dimerization and Stacking in Transition-Metal Bisdithiolenes and Tetrathiolates. J. Am. Chem. Soc. 1985, 107, 6253-6277.

(22) Kresse, G.; Furthmuller, J. Efficient iterative schemes for $a b$ initio total-energy calculations using a plane-wave basis set. Phys. Rev. B 1996, 54, 11169-11186.

(23) Dudarev, S. L.; Botton, G. A.; Savrasov, S. Y.; Humphreys, C. J.; Sutton, A. P. Electron-energy-loss spectra and the structural stability of nickel oxide: An LSDA+U study. Phys. Rev. B 1998, 57, 1505-1509.

(24) Zhou, F.; Cococcioni, M.; Marianetti, C. A.; Morgan, D.; Ceder, G. First-principles prediction of redox potentials in transition-metal compounds with LDA+U. Phys. Rev. B 2004, 70, 235121.

(25) Garreau de Bonneval, B.; Faulmann, C.; Verelst, M.; Lecante, P.; Malfant, I.; Cassoux, P. Structural study and magnetic properties of new $\left[\left(\mathrm{Cp}_{2}{ }_{2} \mathrm{M}\right)_{\mathrm{x}}\left(\mathrm{NiC}_{2} \mathrm{~S}_{4}\right)\right]_{\mathrm{n}}$ polymer salts. Synth. Met. 2003, 133-134, 597-599.

(26) Vogt, T.; Faulmann, C.; Soules, R.; Lecante, P.; Mosset, A.; Castan, P.; Cassoux, P.; Galy, J. A LAXS (large angle $\mathrm{x}$-ray scattering) and EXAFS (extended x-ray absorption fine structure) investigation of conductive amorphous Nickel tetrathiolato polymers. J. Am. Chem. Soc. 1988, 110, 1833-1840.

(27) Faulmann, C.; Cassoux, P.; Vicente, R.; Ribas, J.; Jolly, C. A.; Reynolds, J. R. Conductive Amorphous MetalTetrathiolato Polymers - Synthesis of a New Precursor $\mathrm{C}_{6} \mathrm{O}_{2} \mathrm{~S}_{8}$ and Its Derived Polymers, and LAXS Structural Studies. Synth. Met. 1989, 29, E557-E562.

(28) Tkachov, R.; Stepien, L.; Roch, A.; Komber, H.; Hennersdorf, F.; Weigand, J. J.; Bauer, I.; Kiriy, A.; Leyens, C. Facile synthesis of potassium tetrathiooxalate - The "true" monomer for the preparation of electron-conductive poly(nickel-ethylenetetrathiolate). Tetrahedron 2017, 73, 2250-2254.

(29) Dkhissi, A.; Beljonne, D.; Lazzaroni, R.; Louwet, F.; Groenendaal, L.; Brédas, J. L. Density functional theory and Hartree-Fock studies of the geometric and electronic structure of neutral and doped ethylenedioxythiophene (EDOT) oligomers. Int. J. Quantum Chem. 2003, 91, 517-523. 
(30) Alkan, F.; Salzner, U. Theoretical investigation of excited states of oligothiophene anions. J. Phys. Chem. A 2008, $112,6053-6058$.

(31) Ullah, H.; Shah, A. U. A.; Bilal, S.; Ayub, K. Doping and Dedoping Processes of Polypyrrole: DFT Study with Hybrid Functionals. J. Phys. Chem. C 2014, 118, 17819-17830.

(32) Baughman, R. H.; Brédas, J. L.; Chance, R. R.; Elsenbaumer, R. L.; Shacklette, L. W. Structural Basis for Semiconducting and Metallic Polymer Dopant Systems. Chem. Rev. 1982, 82, 209-222.

(33) Brédas, J. L.; Thémans, B.; Fripiat, J. G.; André, J. M.; Chance, R. R. Highly conducting polyparaphenylene, polypyrrole, and polythiophene chains: An ab initio study of the geometry and electronic-structure modifications upon doping. Phys. Rev. B 1984, 29, 6761-6773.

(34) Brédas, J. L.; Street, G. B. Polarons, Bipolarons, and Solitons in Conducting Polymers. Acc. Chem. Res. 1985, $18,309-315$.

(35) Stafström, S.; Brédas, J. L.; Epstein, A. J.; Woo, H. S.; Tanner, D. B.; Huang, W. S.; MacDiarmid, A. G. Polaron lattice in highly conducting polyaniline: Theoretical and optical studies. Phys. Rev. Lett. 1987, 59, $1464-1467$.

(36) Moliton, A.; Hiorns, R. C. Review of electronic and optical properties of semiconducting $\pi$-conjugated polymers: applications in optoelectronics. Polym. Int. 2004, 53, 1397-1412.

(37) Heeger, A. J.; Sariciftci, N. S.; Namdas, N. B. Semiconducting and Metallic Polymers. Oxford University Press: Oxford, 2010.

(38) Emin, D. Polarons. Cambridge University Press: New York, 2013.

(39) Linares, M.; Hultell, M.; Stafström, S. The effect of lattice dynamics on electron localization in poly-(paraphenylenevinylene). Synth. Met. 2009, 159, 2219-2221.

(40) Cutler, M.; Mott, N. F. Observation of Anderson Localization in an Electron Gas. Phys. Rev. 1969, 181, 13361340 .

(41) Callen, H. B. The Application of Onsager's Reciprocal Relations to Thermoelectric, Thermomagnetic, and Galvanomagnetic Effects. Phys. Rev. 1948, 73, 1349-1358.

(42) Venkateshvaran, D.; Nikolka, M.; Sadhanala, A.; Lemaur, V.; Zelazny, M.; Kepa, M.; Hurhangee, M.; Kronemeijer, A. J.; Pecunia, V.; Nasrallah, I.; Romanov, I.; Broch, K.; McCulloch, I.; Emin, D.; Olivier, Y.; Cornil, J.; Beljonne, D.; Sirringhaus, H. Approaching disorder-free transport in high-mobility conjugated polymers. Nature 2014, 515, 384-388.

(43) Mahan, G. D.; Sofo, J. O. The best thermoelectric. Proc. Natl. Acad. Sci. U. S. A. 1996, 93, 7436-7439.

(44) Madsen, G. K. H.; Singh, D. J. BoltzTraP. A code for calculating band-structure dependent quantities. Comput. Phys. Commun. 2006, 175, 67-71.

(45) Wang, D.; Shi, W.; Chen, J.; Xi, J.; Shuai, Z. Modeling thermoelectric transport in organic materials. Phys. Chem. Chem. Phys. 2012, 14, 16505-16520.

(46) Bardeen, J.; Shockley, W. Deformation Potentials and Mobilities in Non-Polar Crystals. Phys. Rev. 1950, 80, 7280.

(47) Lindhard, J. On the Properties of a Gas of Charged Particles. Mat. Fys. Medd. Dan. Vid. 1954, $28,1-57$.

(48) Li, Q.; Das Sarma, S. Collective excitation spectra of one-dimensional electron systems. Phys. Rev. B 1989, 40, 5860-5863.

(49) Henkelman, G.; Arnaldsson, A.; Jonsson, H. A fast and robust algorithm for Bader decomposition of charge density. Comp. Mater. Sci. 2006, 36, 354-360. 
